\title{
Ethics in Engineering Students' Design Considerations: Case Studies of Elec- tric Power Systems for the "Developing World"
}

\section{Mr. Ryan C. Campbell, University of Washington}

Ryan is a Ph.D. candidate in the University of Washington's interdisciplinary Individual Ph.D. Program. His research interests include: engineering education, ethics, humanitarian engineering, and computer modeling of electric power and renewable energy systems.

Dr. Ken Yasuhara, Center for Engineering Learning \& Teaching, University of Washington

Dr. Denise Wilson, University of Washington

Denise Wilson is a professor of electrical engineering at the University of Washington, Seattle. Her research interests in engineering education focus on the role of self-efficacy, belonging, and other noncognitive aspects of the student experience on engagement, success, and persistence and on effective methods for teaching global issues such as those pertaining to sustainability. 


\title{
Ethics in Engineering Students' Design Considerations: Case Studies of Electric Power Systems for the "Developing World"
}

\begin{abstract}
This work explores engineering ethics empirically in a "developing world" context through a framework of care ethics. Care ethics, a.k.a., the ethic(s) of care, is particularly suitable for the "developing world" context because it helps draw attention to imbalances of power (e.g., inequality, differential opportunity, and limitations on autonomy) that are often neglected by other ethical frameworks. In this work, we selected one element of care ethics (responsibility) and operationalized it in several ways: the language of responsibility; notions of paternalism; and awareness of key, influencing stakeholders. These lenses were developed and refined iteratively by employing them in case study analyses of two design project reports written by teams of senior undergraduate engineering students. Our interpretive analysis of this data outlined significant differences in care-ethical responsibility as viewed through each lens. For example, one group demonstrated little paternalism and considered several key, influencing stakeholders in their report, while the other group employed a more paternalistic approach and considered fewer key influencing stakeholders. In the interest of broadening ethical awareness in engineering, the findings and outcomes of this work can be used by educators to inform the design of course materials, exercises, and evaluation/grading criteria, such as by adapting these lenses for use in self- and/or peer-assessment. This work can also be used by researchers interested in care ethics and/or engineering student responses to issues of humanitarian or social justice nature to inform interview question and survey item design. Finally, this work contributes to the discourse of engineering ethics by developing and applying an ethical framework from the wider literature that has not received much attention in engineering ethics to date.
\end{abstract}

\section{Introduction}

This work explores engineering ethics in a "developing world" context. There are many ethical frameworks with which to examine the involvement of engineers from industrialized countries in projects directed at the "developing world" (e.g., see the Ethics of International Engagement and Service-Learning Project $\left.{ }^{[1]}\right)$. The framework chosen for this study is care ethics, a.k.a., ethic(s) of care (e.g., see Tronto ${ }^{[2,3]}$, Held ${ }^{[4]}$, and Engster ${ }^{[5]}$ ). Care ethics is a normative ethical theory that emphasizes the importance of concern, responsibility, and context, rather than the impartiality and universal standards associated with the more common ethical notions of justice or duty. Care ethics is particularly suitable for the "developing world" context because it helps draw attention to imbalances of power (e.g., inequality, differential opportunity, and limitations on autonomy) that are often neglected by other ethical frameworks ${ }^{[2]}$. This work contributes to the discourse of engineering ethics by exploring the application of an ethical framework from the wider literature that has not received much attention in engineering ethics. For example, a full text search of the journal of Science and Engineering Ethics returns only a handful of relevant papers from 2001 to 2014 that even mention the phrases "ethic(s) of care" or "care ethics," let alone prioritize the subject. 
A few examples of papers that focus directly on care ethics in engineering include the pioneering work of Pantazidou \& Nair ${ }^{[6]}$, who illustrated how care ethics can guide engineering practice by mapping its elements to the processes of engineering design and problem solving. Also, Kardon ${ }^{\text {[7] }}$ used case studies of published engineering failures to align care ethics with an engineer's "standard of care." Finally, Wynsberghe ${ }^{[8]}$ used care ethics to provide normative grounding for a framework and methodology of care-centered value-sensitive design in healthcare robotics.

Examples of other papers that include substantial consideration of care ethics without necessarily making it the central focus include Riley ${ }^{[9]}$, who discussed it within broader considerations of feminism and feminist ethics. Also, Campbell \& Wilson ${ }^{[10]}$ recommended including care ethics in the engineering curriculum via topics of humanitarian engineering. Finally, Sunderland et al. ${ }^{[11]}$ mentioned care ethics in relation to the underappreciated value of emotion in engineering ethics education. The present work extends the current literature by developing concrete, practical tools for exploring care ethics in the context of engineering, and applying these tools to better understand the care-ethical conceptions of future engineers.

\subsection{Description of the Design Project}

In this work, we present a comparative (cross-case) analysis of a selection of design project reports written by senior undergraduate engineering students at a large research university in the western United States. These students were all majoring in electrical engineering, specializing in electric power systems engineering. To complete these projects, groups of three students assumed roles on mock consultant teams to recommend design options that addressed a problem of electro-mechanical dynamic stability in a large-scale electric power transmission system. The three-week project was based on that created by Hines \& Christie ${ }^{[12]}$, which included a realistic model of the western U.S. transmission system and explicitly incorporated technical design, economics, environmental considerations, and societal/ethical considerations. For this research, however, the assignment was modified to situate the project in a "developing world" context in which the students worked with an NGO in a fictitious country (see Figure 1) that had recently re-unified after years of differential "development" (not unlike Germany in 1990 or perhaps Korea sometime in the future). ${ }^{*}$ The electro-mechanical stability problem itself only affected the population living in the industrialized western side of the country, but students were also asked to recommend options for extending the power system into the eastern region in a way that environmental, social, and ethical considerations were taken into account. Students were provided with background materials to enable them to better understand and account for the social and cultural differences in the non-industrialized eastern half of the country. The problem statement below ${ }^{\dagger}$ served as the core prompt, and responses to part (c) are the focus of this paper:

a) Reports should give a description of the transient stability analysis conducted on the included base case system data. This section should include an estimation of the transient stability limit from the present operating levels (given in the enclosed data file [...]). North to South TTC (Total Transfer Capacity) should be calculated by increasing the Northwest generation and reducing Canju generation by the same amount until the system is not transient stable. The difference between the Northwest generation and the Northwest load is the Northwest

\footnotetext{
* Note that even in countries where a reunification situation does not exist, such a context can highlight issues of ethics and social justice that may be present between neighboring countries with wide differences in material wellbeing (e.g., between countries in Western \& Eastern Europe, the U.S. \& Mexico, or South Africa \& Mozambique).

${ }^{\dagger}$ Problem statement adapted from ${ }^{[13]}$.
} 
export and is considered the amount of transfer to Canju. The value of this transfer at which the system is barely stable is the transient stability limit. South to North TTC is similarly calculated.

b) Reports should recommend a North to South allowed TTC plan, and a South to North TTC plan at the CNI (Canju-Northwest Intertie) for each year in the given planning period.

c) Reports should recommend options for extending the system into the eastern region that illustrate how environmental, social, and ethical considerations were taken into account. Note that the population of the eastern region is about half that of the west $(38 \mathrm{M}$ vs $76 \mathrm{M})$ and the [western power] system serves approximately $71 \mathrm{M}$ people.

d) Reports should detail all transmission upgrades recommended. The economic impact of all recommendations should be outlined. The economic data [provided] should be used in any economic analysis of alternatives. System upgrades can be in terms of transmission system controls updates, or transmission line construction. Encouraging new generation capacity in certain areas in addition to the predicted generation growth can be recommended if it is found to be economically justifiable and feasible.

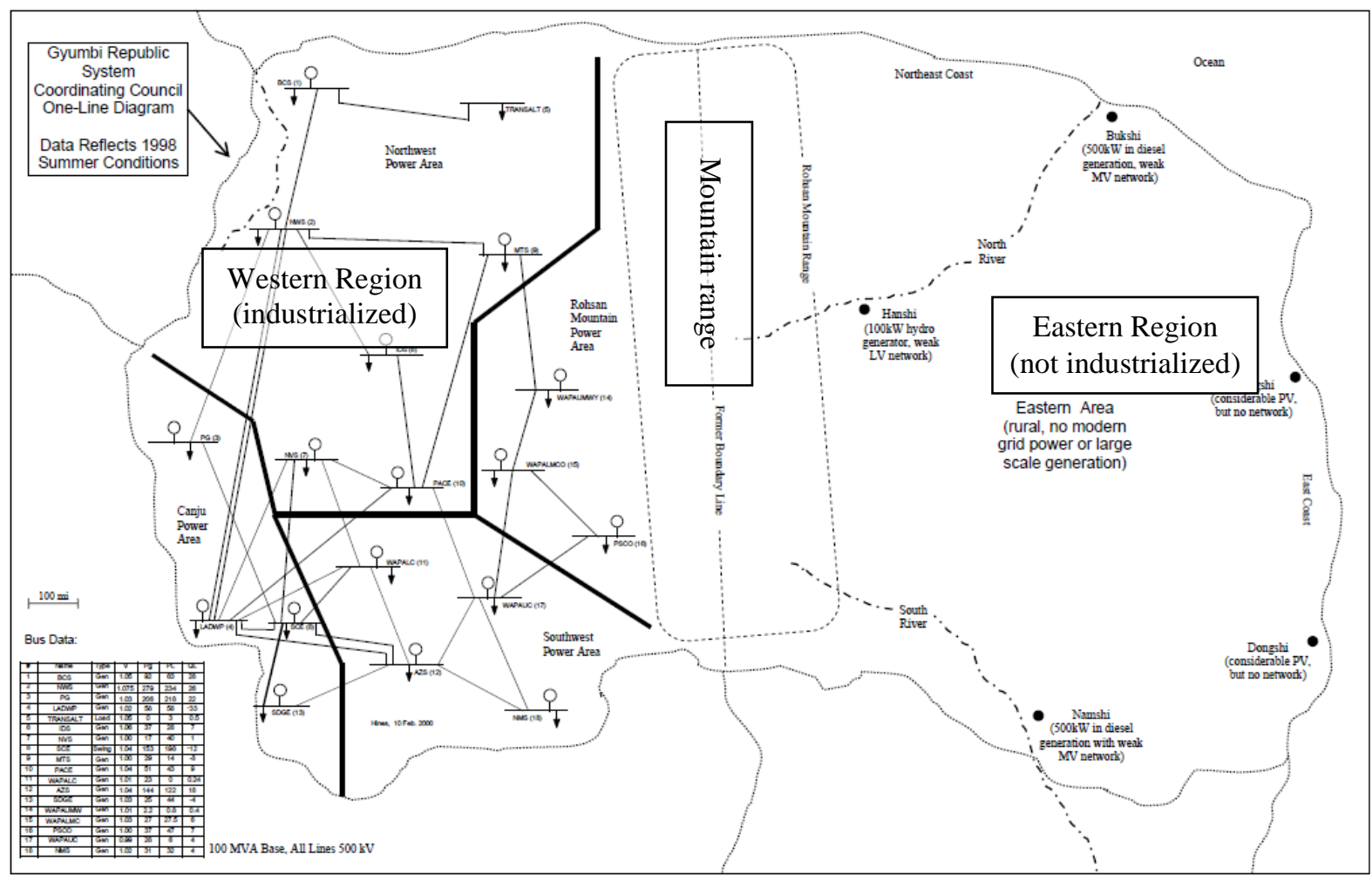

Figure 1. Map* of Gyumbi (a fictitious country)

In addition to the materials referred to in the problem statement above, key contextual information was given, such as the following excerpt of a provided memo:

"The tribal leaders of the Gyumbi Republic...view the process of modernization as a potentially perilous one and wish to proceed in a manner that is both informed of the possibilities and

* Diagram of the western region's electric power system adapted from ${ }^{[13]}$. 
sensitive to the needs and culture of their people. Like many indigenous peoples (such as the Native American and Hawaiian Island people), they treat nature as sacred. They are thus very interested in power system technology that is minimally disruptive to the environment. They have also learned that the approach to power system design employed by much of the developed world is in fact sub-optimal and quite inefficient, because when large-scale generation is far from load centers, there is no way to effectively utilize waste heat."

\section{Ethical Framework*}

In this work we have built upon a framework for care ethics developed by J. Tronto ${ }^{[2]}$. This particular framework has been employed by others for conceptual explorations in engineering ${ }^{[6-8]}$; however, to our knowledge, neither Tronto's framework nor any other framework for care ethics has yet been used as a lens to explore empirical data collected to better understand how care might be reflected in what engineers do and the ways they might think (especially in situations that one might reasonably expect caring qualities to be important, such as in problems of a humanitarian or social justice nature).

Tronto begins by framing care ethics as a practice and notes that there are essentially four phases of any care process as commonly understood: "caring about (noticing the need to care in the first place), taking care of (assuming responsibility for care), care-giving (the actual work of care that needs to be done), and care-receiving (the response of that which is cared for to the care)" [2, p. 127]. These four phases give rise to four moral elements of care: Attentiveness, Responsibility, Competence, and Responsiveness, respectively.

Attentiveness involves awareness of the needs of others and holds neglect and even ignorance (either deliberate or inadvertent, e.g., due to habit or privilege) as moral failings. The next element, Responsibility, is considered central to morality and involves owning one's involvement in the care relationship (be that relationship voluntary or not), deciding whether or not the observed need should be met, by whom, and what action(s) to take. Responsibility here differs from obligation, because it is contextual rather than universal, is more ambiguous, and may or may not even be associated with one's prior actions (i.e., you can choose to be responsible simply because you care and see that no one else is going to help). The next element, Competence, indicates that the work of meeting the need must not only be performed, but it must be done effectively so that the need is actually met. Thus, insincere attempts at care-giving are considered moral failings, as are sincerely well-intentioned but ineffective attempts (thus sharing some alignment with consequentialism). Finally, Responsiveness involves the reaction of the care-receiver to the care provided and includes consideration of the problems of inequality and vulnerability that are present in any caring situation. Responsiveness can be viewed as the feedback loop by which a care-giver can determine if the care provided is accepted by and effective for the care-receiver. This moral element of care challenges the common notion underlying conventional ethical theories that all individuals are equal, self-supporting, and entirely autonomous. The fact that power imbalances exist and that people are unequal, interdependent, and even vulnerable has important implications at both the individual and the societal level.

\footnotetext{
${ }^{*}$ Portions of this section are adapted from ${ }^{[14]}$.
} 
Having described care ethics as a practice, Tronto adds to these four moral elements a fifth metalevel dimension known as the Integrity of Care. The intended connotation of integrity here is cohesiveness, joining together, or integration. Thus, just as good care results from the four phases being well-aligned and collectively appropriate, the four moral elements must fit together as an integrated whole in a way that is sensitive to context and addresses the conflict inherent in any moral situation, be it micro- or macro-ethical, as traditionally understood. The Integrity of Care can also be considered a disposition ${ }^{[15, p .8]}$ that provides a motivational dimension of care ethics, to which the four phases and moral elements add operational "legs" for enacting the process of care. Tronto's framework for care ethics is roughly summarized graphically in Figure 2 and can be described as an interconnected and sometimes overlapping sequence of these five elements.

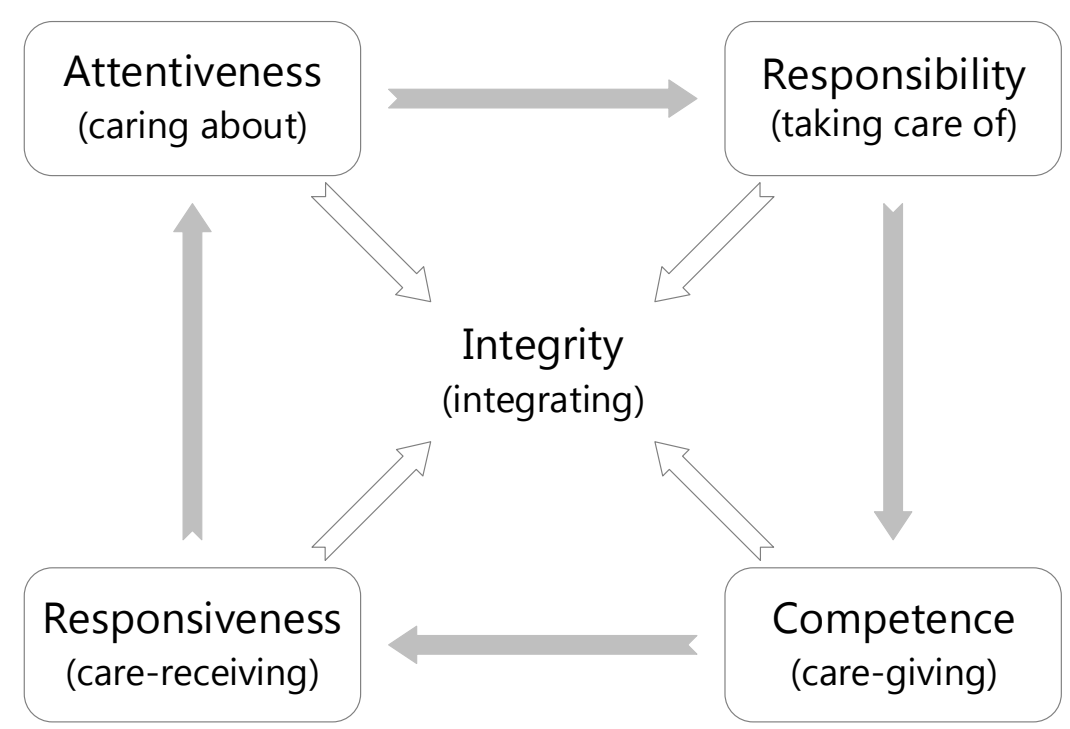

Figure 2. A graphical approximation of Tronto's framework for care ethics

\section{Methods}

In this paper, we have chosen a specific element of care ethics, Responsibility, operationalized" it as described below, and applied it to a selection of the available data.

\section{Language of Responsibility}

The first operationalization of care-ethical responsibility we employ is to look at the language of responsibility, i.e., the ways in which responsibility might be discussed in the group reports. For example, some might use the term "responsibility", others the term "obligation", or they may simply talk about what "should" (or "could") be done. In the context of engineers helping others in a developing world scenario, all of these varying ways of discussing responsibility could have something to say about care-ethics. For example, variations in wording may be indicative of commitment levels or notions of agency.

\footnotetext{
${ }^{*}$ We use the term operationalize here somewhat loosely in that our purpose in operationalizing is not really to measure but to improve our understanding of the concept of care-ethical responsibility. While measurement and/or assessment may also be possible, this is not the primary objective.
} 


\section{Paternalism}

The second operationalization of care-ethical responsibility we employ looks at the level of re-

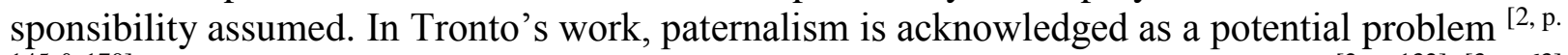
$145 \& 170]$ and has been associated with the notion of taking too much responsibility [2, p. 132], [3, p. 63]. Thus, paternalism is essentially a symptom of imbalance in the assumption of Responsibility. Paternalism involves a care-giver assuming he/she knows what is best for the care-receiver and making decisions that circumvent their wishes for their own good. This may not seem like a problem in some situations, such as when keeping a toddler from running into the street. In fact, this assumption of power may be acceptable and even desirable when care-receivers (be they humans, animals, or other living things) are incapable of making their own decisions or lack the knowledge or education necessary to understand the consequences of their intended actions. However, the danger of paternalism is that it can rob other people of their rights, destroy their sense of independence, and create long-term patterns of dependency and privilege.

\section{Stakeholder Awareness}

The third and final operationalization of care-ethical responsibility we employ involves stakeholder considerations. For the purpose of this work, stakeholders are broadly defined as those entities that would influence or be affected by the eastern expansion of the power system. Note that the terms "influencing" and "affected by" are used intentionally to avoid confusion (e.g., "affecting" vs. "affected" would be harder to distinguish). When deciding what to do about a situation, assuming care-ethical responsibility well requires knowledge of one's own strengths and abilities, as well as awareness of others who might be better positioned to assist. This helps ensure that decisions about anticipated actions are not naïve or unrealistic. From the perspective of Tronto's first element of care ethics (Attentiveness), consideration of stakeholders who are affected by the situation (especially those who are vulnerable, powerless or otherwise underprivileged) can be thought to carry more weight than consideration of the influencing stakeholders, who are better positioned to take care of themselves. However, from the perspective of careethical responsibility, we suggest that considerations of the influencing stakeholders are more the focus, because assuming responsibility is an active process undertaken by people who choose to influence the situation. This is not to say that, when assuming care-ethical responsibility, one should set aside or forget about affected stakeholders. Indeed that would be contrary to the spirit of care ethics, which espouses an integrated and holistic approach, as Tronto's Integrity of Care reminds us (e.g., see Figure 2, above). As a first-order estimation of care-ethical responsibility, we could examine, for example, the number or breadth of influencing stakeholders considered.

Using the above three lenses under an interpretive conceptual framework, we employed qualitative methods of case study analysis in this work. ATLAS.ti qualitative data analysis software was used to manage and mark up the reports, which enabled analysis to occur in the full context of the written reports (rather than excising parts to be examined out of context) and thereby minimized misinterpretation. Our analysis focused on student responses to the issue of expansion into the non-industrialized eastern region of the country (see Section 1.1).

\subsection{Case selection}

The reports analyzed in this paper were written by senior undergraduate students at a large research university in the western United States. All students were majoring in electrical 
engineering and specializing in electric power systems. Students formed self-selected groups of three to complete the design projects and write up the reports. A total of 36 students were enrolled in the course, including 7 women and 29 men. Other demographics, such as race/ethnicity, were not collected for this class because it was not clear at the time if student work would be used for research purposes. However, in the interest of fair and unbiased grading, the reports had been anonymized shortly after receipt. IRB permission was requested some time later for their use in research. Consequently, analysis was performed without any knowledge of the gender breakdowns or other characteristics of the group members.

To aid in the selection of cases to analyze in detail, word counts were performed of both the entire reports and the selections of passages identified as being pertinent to eastern expansion (coded "east-side addressed"). As shown in Figure 3, the word counts reveal something of the varying degrees to which the student groups engaged in the eastern expansion aspect of the project, with some groups writing approximately 2,000 words on the topic and others writing only about 200 (see left axis and bar chart in Figure 3). Normalizing by total word count, this corresponded to a range of $38 \%$ to $7 \%$ of the non-numerical content of the reports (see right axis and line chart in Figure 3). The two cases chosen are referred to in this paper as Rose and Lilac*. These reports were selected for analysis because they (1) exhibited contrasting approaches to eastern expansion, (2) differed in the apparent importance they placed on eastern expansion as indicated by the absence or presence of eastern expansion in the executive summary, and (3) provided the most material of interest for analysis. The selection of these two cases also attempts to minimize the effect of the varying levels of engagement with the east-side expansion.

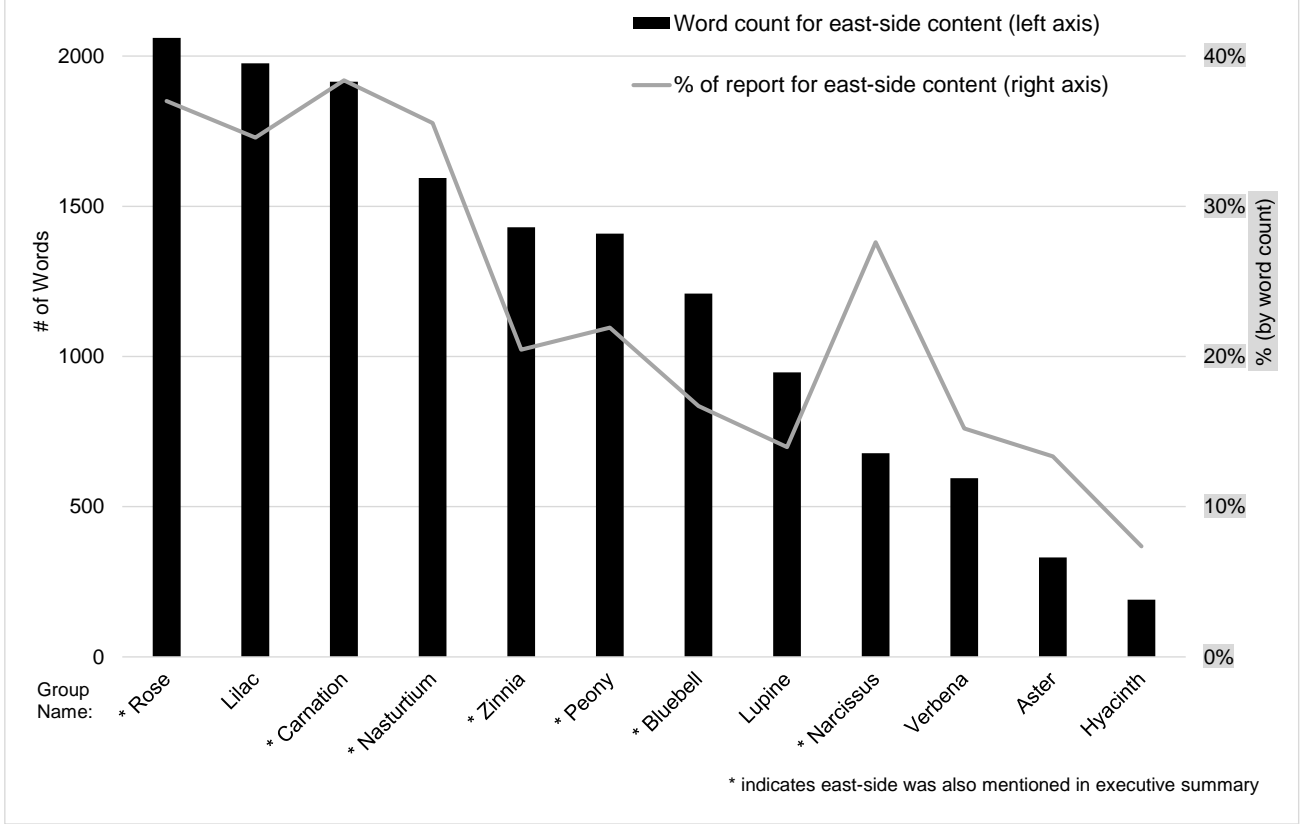

Figure 3. Word counts used to identify cases for detailed analysis

\footnotetext{
* Code names, rather than numbers or letters are used in this work to avoid implying order or rank. The names used in this analysis were inspired by a convention in the electric power industry for identifying overhead electrical conductor types using code words such as plant and bird names instead of alpha-numerical codes. Thus, the student groups in this work were randomly assigned names, in this case the names of flowers from page 7 of ${ }^{[16]}$.
} 


\section{Findings \& Discussion}

In this section, we describe relevant selections from each of the reports with regard to careethical responsibility as operationalized in the Methods section. Due to variations in the way these concepts appeared in the two reports, the order of their presentation varies below.

Quotations used from the reports are numbered for ease of reference. We conclude the section with a comparison of the findings between the two teams' reports.

\subsection{Team Rose}

In this section, we present the findings from analysis of Team Rose's report, which contained over 2,000 words pertaining to the eastern expansion (37\% of their total report). We begin with the language of responsibility, followed by indications of paternalistic/non-paternalistic approaches, and end with stakeholder awareness.

\section{Language of Responsibility (Team Rose)}

Team Rose wrote explicitly about responsibility in a number of senses, including that held by the western region toward the eastern region and that assumed by themselves as consultants. With regard to the latter, they espoused an active and explicit stance on group responsibility for their work in the eastern region when they wrote the following in the introduction of their report:

"We believe that it is our responsibility to provide guidance for a system in this region that preserves [the] traditions and culture of the area, but also bring [s] all the benefits of electrification, including improvements for health, education and wellbeing."

This quote demonstrates a clear and specific level of commitment "to provide guidance" and suggests a sense of agency in their ability to do so in a manner that is culturally sensitive and respectful. This quote will be further explored in the Paternalism subsection below.

Another sense of responsibility found in Team Rose's report was that held by the western region toward the eastern region. About this they wrote,

"...providing support from the Western Region is socially responsible, as the Western region has a modern power infrastructure. The reunification necessitates that Western Gyumbi provide some of its significant resources to the Eastern side in order to help develop the country."

In terms of ethics, this invocation of social responsibility could be interpreted to focus on justice or duty rather than on care, but note that, as Engster ${ }^{[5]}$ suggested with the title of his book The Heart of Justice, the underlying motivation for fairness is essentially caring: if we did not care about others (and/or ourselves), then why would it matter if one group of people had resources that others did not, or had license to behave in ways that others did not? In fact, care ethics goes beyond the notion of equality presumed by common ethical notions of justice, fairness, or duty and draws attention to the fact that, in many cases, people are not and cannot be equal because they have different opportunities, privileges, and needs. ${ }^{*}$ While the above quote does evoke

\footnotetext{
* Note that Tronto associated these issues of difference/privilege with the Responsiveness element of care ethics,

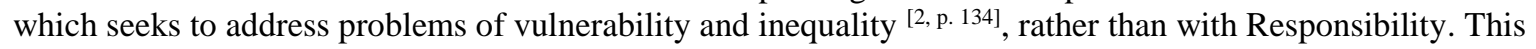
shows the interconnected nature of care ethics and how multiple elements might overlap.
} 
notions of fairness and duty (e.g., that the western region should share its technology and resources with the eastern region because they are now a unified country), note the use of the phrase "to help," which demonstrates a caring impulse that goes beyond fairness or duty. This phrase, in conjunction with the preceding phrase, "provide some of its significant resources," shows recognition of the difference in opportunity enjoyed by the western region. These can be considered evidence of care-ethical responsibility.

Later in the report, Team Rose reiterated the idea of social responsibility with the following:

"The reunification of Gyumbi brings increased importance to the development of the Eastern Region's electric infrastructure. As a single country, political leaders and policy makers have an obligation to build a power system on the east side that fully considers environmental, social and ethical factors."

Here they use the term "obligation" as held by specific stakeholders. "While Tronto draws a distinction between obligation and responsibility (see the third paragraph of Section 2 above), common usage of the terms does not, and most dictionaries and thesauruses list responsibility as a synonym for obligation. Team Rose's association here of obligation with considerations of social and environmental factors could be interpreted as evidence for awareness of the needs of others (human or otherwise), and thus as care-ethical attentiveness, which is a precursor to careethical responsibility.

\section{Paternalism (Team Rose)}

Quote (1) above, which focused on Team Rose's responsibility as a consultant, provided an example of interacting with others in a non-paternalistic manner. In terms of care-ethical responsibility, this quotation demonstrated an approach to assuming responsibility that appeared sensitive to cultural differences and sought to avoid paternalistic decisions that could even destroy those differences. The phrase "provide guidance" suggested awareness of the role they were playing as advisors rather than designers for the eastern people. Similarly, the phrase "preserves traditions and culture" showed awareness of the significance of the way in which that guidance would be given. In fact, following quote (3) above (regarding the obligations of political leaders and policy makers), they wrote,

"Many of the traditions and culture of Eastern Gyumbi population are vastly different than citizens of Western Gyumbi. It is important that these lifestyles are preserved, along with providing much greater access to the benefits of electrification (i.e. increased healthcare and education)."

These two quotations support the assertion that Team Rose was striving to avoid the danger of paternalism in their work. Also, while not the focus of this paper, it is interesting to note that quote (4) demonstrates care-ethical attentiveness to the needs of the eastern people (i.e., preservation of their culture/lifestyle, as well as their healthcare and education).

\footnotetext{
* This quote will be further explored in the Stakeholder Awareness sub-section below.
} 


\section{Stakeholder Awareness (Team Rose)}

A number of specific stakeholder groups were mentioned in the quotes above, several of which were indicated as influencing the situation. In quote (1), Team Rose indicated themselves, writing about their own role as technical advisers to provide guidance in a culturally sensitive manner. This demonstrated care-ethical responsibility in that awareness of one's own role and how it is best performed are prerequisites for determining appropriate actions to take (or not to take).

Care-ethical responsibility can also be exhibited through awareness of other stakeholder groups. For example, in quote (2), Team Rose wrote about the western region (meaning its people and perhaps government collectively) and the positive influence it could have "to help develop the country." In quote (3), they wrote of the obligation held by political leaders and policy makers to consider environmental, social, and ethical factors. This demonstrated awareness of the power and influence these stakeholders have to impact both people and the natural environment through the consequences of their decisions. The stakeholders indicated in both of these quotes are evidence of care-ethical responsibility in that they demonstrate knowledge of the roles and abilities of others, which helps ensure that anticipated actions are not naïve, unrealistic, or incompatible.

In quote (4), Team Rose mentioned the citizens of the western region; however, in this case it was not clear if they were invoked solely as a point of comparison with the differing cultures in the eastern region, or if they comprised a potentially influencing stakeholder group. If the latter were the case, then the quote may suggest awareness of the danger of cultural imperialism, which could occur if the western region's culture (either by accident or intention) were to accompany expansion of its electric power system. This too then would show care-ethical responsibility through awareness of the potentially negative influence of this stakeholder group.

Systematic analysis of all stakeholders mentioned by Team Rose revealed a number of additional (and sometimes overlapping) stakeholder groups/entities. However, the only influencing stakeholders found were those mentioned above (i.e., political leaders, policy makers, the people/government of the western region, Team Rose themselves as consultants, and perhaps citizens of the western region). Regarding affected stakeholders mentioned, which would be considered indications of care-ethical attentiveness rather than responsibility, these included electric power customers, the farming and fishing industries, the economy, population centers, cities in the east side, residents of the east side, and residents of the entire country.

\subsection{Team Lilac}

In this section, we present the findings from analysis of Team Lilac's report, which contained over 1,900 words pertaining to the eastern expansion (35\% of their total report). Due to the way the care-ethical considerations of interest intersect with the structure of the report, we begin this time with stakeholder awareness, followed by language of responsibility, and end with indications of paternalistic/non-paternalistic approaches. 


\section{Stakeholder Awareness (Team Lilac)}

Systematic analysis of Team Lilac's report revealed awareness of several influencing stakeholders and many affected stakeholders. Most of these occurred in a section of the report labeled "Environmental Impacts." This section considered transmission line impacts and suggested mitigation strategies for a variety of potential issues, including those associated with aesthetics, agricultural lands, archeological/historical sites, endangered and protected species, property owners, recreation areas, waterways, wetlands, and woodlands. Undergraduate engineering students would not normally be expected to possess such considerable knowledge of these various issues and the associated stakeholder groups, but in this case the students discovered a reference document published by a U.S. state public service commission specifically on the subject of the environmental impacts of transmission lines (see ${ }^{[17]}$ ), which clearly aided them in structuring and writing this section of their report.

Two influencing stakeholder groups that appeared in Team Lilac's report were archeological and historical preservation societies/organizations. These were mentioned in association with responsibility (to be discussed further in the next subsection) for protecting sites with archeological/historical value. Another group that was discussed in terms of its influence was farmers who would work with others (presumably engineers who were not explicitly mentioned) on the placement of poles/towers to mitigate impacts on farm operations and/or agricultural lands. These two instances demonstrated care-ethical responsibility by showing knowledge of potentially overlooked stakeholder groups who might have a special role to play (i.e., providing the requisite knowledge and capability of identifying historically important areas in the first case and in optimizing farm operations in the second).

Another influencing stakeholder group that Team Lilac mentioned was the electric power utility who would be involved in compensating owners of private property for the use of their land. On this they wrote,

"If the path of the lines needs to cross private property, negotiations about fair payments need to be made between land owners and utilities to compensate for the negative impacts."

In this case, the relevance of this consideration to the eastern expansion scenario is questionable, given that the concept of private property may not even exist in the tribal lands. However, if we focus on the influencing stakeholder (rather than what appears to be a misidentified affected stakeholder ${ }^{*}$ ), this quote also shows care-ethical responsibility through awareness of the influence of the electric power utility and its obligation to fairly compensate affected stakeholders.

\footnotetext{
* Team Lilac explicitly indicated that the environmental impacts section of their report was intended for considerations of the Eastern expansion scenario. However, given the source of the information it contained and the industrialized audience for whom that source was originally written, this section of the report sometimes appeared to better cover considerations of transmission line construction in the industrialized western region (i.e., since adding a supplemental transmission line was suggested as a solution to the stability problem of the western region). None-the-less, many of these stakeholder considerations could have been conceptually extended to consider native inhabitants and more culturally appropriate means of addressing their concerns. Unfortunately, Team Lilac did not make the effort to adapt these industrialized-world considerations to the context at hand. This idea will be further explored under the Paternalism section below.
} 
Systematic analysis of all stakeholders mentioned by Team Lilac revealed a number of additional (and sometimes overlapping) stakeholder groups/entities. However, the only influencing stakeholders found were those mentioned above (i.e., archeological and historical preservation societies/organizations, electric power utilities, and farmers). It is perhaps noteworthy that this team did not explicitly indicate themselves as influencing stakeholders (more on this in the next section). Regarding affected stakeholders mentioned, which would be considered indications of care-ethical attentiveness rather than responsibility, these included owners of private property, users of recreation areas, farms/farmers (who were indicated in both directions of influence), endangered and protected species of plants/animals, and the plants/wildlife/ecosystems associated with waterways, wetlands and woodlands.

\section{Language of Responsibility (Team Lilac)}

Team Lilac explicitly referred to responsibility only once in their report. This occurred in connection with a suggestion to mitigate concerns over preserving locations of archeological or historical value. Specifically, they suggested finding

"...organizations or societies in that location that are responsible for protecting archeological and historical sites so that information can be found about whether a[n archeological] survey needs to be done at the construction site."

In this quote, responsibility was ascribed to others outside the engineering-consultant role of the group. (Note: Care-ethical considerations due to stakeholder awareness evinced in this quote were discussed in the previous section.)

Another way responsibility was discussed in Team Lilac's report was through use of the word "should" when discussing issues related to others' responsibilities, such as that of financial burden:

"The social and ethical issues that affect this extension project are the argument as to who should incur the construction cost."

Here the phrase "who should incur" could easily be replaced with the phrase "who should be responsible for" and would suffer no change in meaning. Thus, responsibility is invoked in a conventional sense of duty or obligation. They then continue,

"The population of the eastern region is half that of the western and they are a less developed area so these people lack the capability to generate the revenue required to undertake the construction of the transmission lines. It would not be fair to raise rates in the western region in order to pay for an expansion project, which primarily benefits the Eastern Area."

This quote clearly focuses on the issue of justice or fairness, and while the relative financial disadvantage of the eastern region is acknowledged, this does not appear to matter from the perspective of fairness. However, the following sentence appears more care-ethically oriented with this concession:

"From an ethical perspective, it would be wrong to leave 1/3 of the Gyumbi population without a reliable interconnected source of power so minor construction projects should be made in the future." 
The word "should" in the quote above serves as a proxy for responsibility without clearly identifying the responsible party. In this case, their use of the word is consistent with their role as consultants, and the responsible party is understood to be their client or perhaps the governmental agencies and other decision-makers with whom the report would likely be shared. Looking at the above three quotes together shows that this group conceives of ethics as something more than just justice or fairness and has perhaps an intuitive* sense that differences in power, opportunity, and privilege need to be somehow taken into account. Care ethics would have provided them a helpful framework in this regard if it had been provided (see Section 5.1) or were more easily found in an Internet search targeting engineering ethics.

Finally, it is worth noting that Team Lilac made no explicit statements of their own group's responsibility for the eastern expansion. This could suggest a low level of commitment to assuming responsibility for this aspect of their work. In contrast, there were several explicit indications of Team Lilac as an influencing stakeholder in the solution to the western region's stability problem. An alternative explanation for this difference (beyond, or in addition to, a low level of commitment to the eastern expansion portion of the project) might be variations in writing style, as it is possible that different team members wrote each these sections.

Nevertheless, there are indications of implicit assumption of responsibility for their role as consultants in the eastern expansion. These appeared in the report as preliminary design options they explored for expansion into the eastern region ${ }^{\dagger}$ and as mitigation strategies they suggested to address potential concerns over constructing new power transmission infrastructure (see the Stakeholder Awareness section above).

\section{Paternalism (Team Lilac)}

As suggested in a footnote of the Stakeholder Awareness section for Team Lilac above, some of their suggestions for mitigation strategies appeared lacking in care-ethical responsibility due to a lack of cultural sensitivity. This can easily lead to paternalism, through which the engineer assumes too much responsibility and usurps the freedom of those they are attempting to help. While many of Team Lilac's stakeholder considerations could be conceptually extended to consider native inhabitants and more culturally appropriate means of addressing their concerns, they did not make the effort to adapt many of their industrialized-world considerations to the context at hand. Quote (5) above was an example of this, given that the concept of private property may not even exist in the non-industrialized tribal lands. Another example was the suggestion to mitigate concerns over visual aesthetics using

"...vegetation such as trees or bushes [that] could be used to block the view of the lines and poles so that they become less intrusive."

This suggestion seems to miss the point that the people in the eastern region view nature as sacred. Simply hiding the environmental damage caused by clearing a transmission line's rightof-way fails to address the core problem of harming nature in the first place and could show

\footnotetext{
* A statement near the end of the report indicated that this group struggled with understanding the definition of social and ethical issues, tried to look online for information, but found nothing useful due to time constraints. Then they wrote: "As a result, common knowledge on what our interpretations was used to answer the question."

$\dagger$ Team Lilac provided line drawings indicating (1) a loop/ring system connecting all the eastern cities with the two nearest western generation sites and (2) a radial system extending from the nearest western generation site to each eastern city via separate laterals off the main line.
} 
disregard for the beliefs of the people. Such an approach to technological and economic development would be paternalistic (i.e., too much responsibility being assumed by the engineers, ostensibly for the good of the people). Evidence of paternalistic thinking was even articulated by Team Lilac in the following quote, which directly followed quote (9):

"Even though the indigenous people in the eastern region view modernization as an invasion into Mother Nature, improving power transmission [in] that area aids in future development \& prosperity of the Eastern region."

This quote seems to suggest that a modern electric power system like that of the western region (and that of most industrialized nations today) should eventually be forced on the eastern region to ensure development and prosperity in spite of the religious beliefs of the indigenous eastern people. Apparently, Team Lilac did not explicitly consider alternative approaches to electrification (some of which were even suggested in the project materials) that could be possible under the social/environmental constraints given for the eastern region.

\subsection{Cross-case Comparisons}

As a first-order estimation of the relative levels of care-ethical responsibility exhibited in these reports, we could compare the number and breadth of relevant stakeholders considered. Limiting the analysis to clear indications of both stakeholders and their influence, we see that Team Rose considered five stakeholder groups (i.e., political leaders, policy makers, the people via the government of the western region, and Team Rose themselves as consultants). For Team Lilac, we found four (i.e., archeological and historical preservation societies/organizations, electric power utilities, and farmers). However, the difference in these numbers is not significant, especially given that additional stakeholder groups/entities were implied in both reports. Rather than expanding the analysis to include implied stakeholders (which could involve more challenging and subjective interpretations), we choose instead to look at the breadth of these explicitly mentioned influencing stakeholders. Here we see that Team Rose mentioned more key, primary stakeholders (e.g., themselves, political leaders, policy makers), while Team Lilac included more secondary or peripheral stakeholders (e.g., farmers, archeological and historical preservation societies/organizations). In terms of responsibility (care-ethical or otherwise), it is clearly important to fully consider the key, primary stakeholders, as without them the actions of the secondary stakeholders may be insignificant. Team Rose thus had the more care-ethically responsible considerations in this regard.

Another way to compare levels of care-ethical responsibility in these reports is to look at the language of responsibility. Unlike Team Rose, who wrote explicitly of assuming responsibility for the eastern expansion aspect of the project, Team Lilac made no explicit indications in this regard. Clearly an articulated, self-aware approach like that of Team Rose is preferable to, and more likely to yield good results, than the implicit (and possibly unexamined/unconscious) approach of Team Lilac. Additionally, Team Rose's report espoused a much lower level of paternalism than that of Team Lilac. While there is much to learn from both reports, Team Rose's was more care-ethically responsible across all three measures.

\footnotetext{
* In terms of care-ethical attentiveness, however, Team Lilac's list of affected stakeholders is clearly the stronger of the two (see the last sentence of each group's Stakeholder Awareness sections above).
} 


\section{Conclusions}

In this work, we applied care ethics - an ethical framework that is seldom invoked in engineering - to a selection of project reports written by electrical engineering students in a senior capstone design course at a large research university in the western United States. The three-week project involved recommending design improvements to an existing large-scale electric power transmission system and explicitly incorporated technical design, economics, and consideration of environmental, societal, and ethical issues. The project was contextualized in a "developing world" setting and highlighted issues of ethics and social justice that are present between neighboring counties with wide differences in material well-being.

In this paper, we adopted a conceptualization of care ethics from the wider literature ${ }^{[2]}$ and focused on a single element of that framework: care-ethical responsibility. We then proposed three operationalizations of this element and used them as lenses for case study analyses of the reports from two student groups. While case studies are not intended to imply prevalence of findings or lead to widely generalizable conclusions, they can uncover things that might otherwise remain hidden and suggest questions for further inquiry. The case study approach provided the opportunity to examine the data in more depth and supported development at the conceptual level that would be considerably more difficult (if not impossible) using other methods. This proved to be a useful means of bridging between the empirical and conceptual aspects of the work, which, while presented in the standard IMRD (Introduction, Methods, Results, Conclusions) order, were actually co-developed iteratively, with the ideas in the methods section both constraining and being modified by the findings over the course of analysis and paper development.

Our findings showed differences in the way responsibility was discussed, which may be indicative of varying levels of commitment and/or notions of agency. Further exploration, such as explaining why these differences exist and what they might mean, is suggested as future work. We also found contrasting approaches to design in the developing world context, with one group demonstrating awareness, sensitivity, and appreciation of the expressed needs of the end user, and the other group adopting a more paternalistic approach suggestive of technological imperialism.

\subsection{Implications for Educators and Researchers}

The development of the operationalizations of care-ethical responsibility used in this work and their application to the analysis of these student reports was performed several years after the reports themselves were written. Thus, there was no opportunity to provide feedback to those students on the care-ethical strengths and opportunities for improvement of their work. However, the ideas and lenses presented here can be used by other educators (and will be used by the authors) in future courses to inform the design of course materials, exercises, and evaluation/ grading criteria. For example, students could be asked to perform assessments of their own work or that of their peers to examine the language of responsibility or the ways in which responsibility was discussed (e.g., implicitly vs. explicitly, or as compulsory/obligatory vs. voluntary/aspirational). They could also identify numbers and types of stakeholders considered and whether or not these stakeholders show care-ethical responsibility (e.g., reflective selfawareness of the engineer's own role, and other important stakeholders who should be called on for their abilities and expertise) or care-ethical attentiveness (e.g., awareness of vulnerable 
stakeholders who are affected by the situation). Similarly, students could self- or peer-assess for presence (or levels) of paternalism.

The operationalizations and findings from this work can be used to inform future research, such as the development of interview questions and/or survey items designed to capture engineering student responses to issues of humanitarian or social justice nature. Only through the appropriate use of larger sample sizes and validated, reliable instruments can questions of cause and effect be answered and generalizable conclusions be reached. This work provides some of the necessary first steps toward understanding the concepts and constructs needed to begin larger-scale investigations into this area of research. In terms of conceptual/philosophical research, this work similarly provides some first steps, empirically grounded in an engineering context, from which to explore how care ethics might be applied to engineering or how engineering might need to change to become more consistent with care ethics.

Another possible implication of this work is directed more broadly at the engineering curriculum. If the paternalistic approach to design exhibited by Team Lilac above proves to be common (and preliminary analysis of the other data from this course suggests that other groups used similar approaches), then this suggests that concepts of design that are user-centered and participatory should be formally introduced in more disciplines, as they are largely unknown in power engineering and mainstream electrical engineering. Participatory practices should occur early and consistently in the design process to maximize their effect. Also, waiting until a senior design project to introduce engineering students to what some people might consider an ethical responsibility to design for the user is clearly not ideal; such considerations should occur much earlier in the curriculum. ${ }^{*}$

\section{Bibliography}

[1] Ethics of International Engagement and Service-Learning Project. (2011). Theoretical Frameworks. In EIESL Web-Based Guidebook. Vancouver, BC, Canada. Retrieved from http://ethicsofisl.ubc.ca/?page_id=37

[2] Tronto, J. C. (1993). Moral Boundaries: A Political Argument for an Ethic of Care. London, Great Britain: Routledge.

[3] Tronto, J. C. (2013). Caring Democracy: Markets, Equality, and Justice. New York, USA: New York University Press.

[4] Held, V. (2006). The Ethics of Care: Personal, Political, and Global. New York, NY, USA: Oxford University Press.

[5] Engster, D. (2007). The Heart of Justice: Care Ethics and Political Theory. New York, NY, USA: Oxford University Press.

[6] Pantazidou, M., \& Nair, I. (1999). Ethic of Care: Guiding Principles for Engineering Teaching and Practice. Journal of Engineering Education, 88(2), 205-212.

\footnotetext{
* Many thanks to the anonymous abstract reviewer who suggested this.
} 
[7] Kardon, J. B. (2005). Concept of "Care" in Engineering. Journal of Performance of Constructed Facilities, 19(3), 256-260. http://doi.org/10.1061/(ASCE)0887-3828(2005)19:3(256)

[8] Wynsberghe, A. van. (2013). Designing Robots for Care: Care Centered Value-Sensitive Design. Science and Engineering Ethics, 19(2), 407-433. doi:10.1007/s11948-011-9343-6

[9] Riley, D. (2013). Hidden in Plain View: Feminists Doing Engineering Ethics, Engineers Doing Feminist Ethics. Science and Engineering Ethics, 19(1), 189-206. doi:10.1007/s11948-011-9320-0

[10] Campbell, R. C., \& Wilson, D. (2011). The Unique Value of Humanitarian Engineering. In Proceedings of the American Society for Engineering Education (ASEE) Annual Conference (pp. 1-11). Washington DC, USA: American Society for Engineering Education.

[11] Sunderland, M. E., Ahn, J., Carson, C., \& Kastenberg, W. (2013). Making Ethics Explicit: Relocating Ethics to the Core of Engineering Education. In Proceedings of the American Society for Engineering Education (ASEE) Annual Conference. Washington DC, USA: American Society for Engineering Education.

[12] Hines, P. D., \& Christie, R. D. (2002). A Capstone Design Project to Meet the Needs of the Changing Power Systems Industry and Satisfy New Accreditation Standards. IEEE Transactions on Power Systems, 17(3), 535 542. doi:10.1109/TPWRS.2002.800869

[13] Hines, P. D. (2001). A Power Systems Capstone Design Project and Associated Simulation Software Designed to Meet the Changing Needs of the Electrical Power Industry and Engineering Accreditation Requirements (Master's Thesis, pp. 84-86). University of Washington, Seattle, WA, USA.

[14] Campbell, R. C. (2013). How Can Engineering Students Learn to Care? How Can Engineering Faculty Teach to Care? In J. Lucena (Ed.), Engineering Education for Social Justice: Critical Explorations and Opportunities (Vol. 10, pp. 111-131). Dordrecht, Netherlands: Springer.

[15] Hawk, T. F. (2011). An Ethic of Care: A Relational Ethic for the Relational Characteristics of Organizations. In M. Hamington \& M. Sander-Staudt (Eds.), Applying Care Ethics to Business (Vol. 34, pp. 3-34). Dordrecht, Netherlands: Springer.

[16] The Aluminum Association, Inc. (1999). Code Words for Overhead Aluminum Electrical Conductors. Arlington, VA, USA. Retrieved from http://www.aluminum.org/sites/default/files/Code\%20Words\%20for\%20Overhead\% 20Aluminum\%20Electrical\%20Conductors.pdf

[17] Public Service Commission of Wisconsin. (2009). Environmental Impacts of Transmission Lines. Madison, WI, USA. Retrieved from http://web.archive.org/web/20090519211144/http://psc.wi.gov/thelibrary/publications/electric/electric10.pdf 